\title{
A Research: Hyperspectral Image Processing Techniques
}

\author{
Kiran Gowda C, S Usha, C J Jagadeesha
}

\begin{abstract}
Hyperspectral image contains more information which are gathered from numerous narrow wavebands from one or more regions, and large amount of data are huddled. An basic problems in hyperspectral image processing are dimension reduction, target detection, target identification, and target classification. In this document, we reviewed the latest activities of target classification, most frequently used techniques for dimension reduction, target detection. Hyperspectral image processing is a complicated process which rely on mixed agents. Here we also recognized and reviewed problems faced by some methods and to overcome the problems, current techniques are discussed and highlighted good methods. To improving correctness, genuine classification techniques and Detection Techniques analysis are recommended.
\end{abstract}

Keywords - Dimensionality Reduction(DR); Hyperspectral Image(HSI); Independent Component Analysis(ICA); Principal Component Analysis(PCA); Projection Pursuit(PP); Target $\operatorname{Detection}(T D)$;

\section{INTRODUCTION}

Hyperspectral data is classified as Feature selection/extraction followed by Information extraction, both of these might be either supervised or unsupervised[13]. The un-supervised methods recognizes the sample of interest contained by an image data. This set of methods do not prefer prior knowledge where as supervised methods make use of prior knowledge on target characteristics whereas should have some knowledge selecting. Although there are several unsupervised methods, presently the most commonly used technique are been conveyed here.

\section{A. Projection Pursuit (PP)}

The greater number of methods with the detection of targets requires an analytical models as precarious mixtures, the method that is employed to plan an dataset of higher dimensional into lower dimensional space of data by preserving the significant data. As the targets were very small when compared to the respective backgrounds, the particular targets were designed which caused the outliers of respective background distribution. To make out the finest projections, an enhanced Projection Pursuit evolutionary algorithm (PPEA) is considered in target detection because of introducing a new technique zero-detection thresholding [14]. A novel method Legendre index for anomaly detection is considered based on projection pursuit, the designed PP technique is capable in detecting anomalies with the quantity of segregating with this frequent classification

Revised Manuscript Received on July 18, 2019. India

Kiran Gowda C, Research Scholar, CSE, RRCE, VTU, Karnataka,

S Usha, RRCE, VTU, Bangalore, Karnataka, India.

C J Jagadeesha, RRSSC(ISRO), FIE, IEI-KSC, Bangalore, Karnataka, India. described with the gray level of a pixel. Reed-Xiaoli (RX) method is applied in addition with PP to isolated outliers[15].

\section{B. Principal Component Analysis(PCA)}

PCA having more than one variable in general which is designed for reducing data redundancy and dimensionality[13].

Authors identified that, intrinsic dimensionality can be successfully reduced in hyperspectral data by using hierarchical PCA algorithm. In this, images are split into different parts, applied PCA on each part later the results are merged. The obtained results are same as the results of traditional PCA. Due to this analysis, it known that classification accuracy is too close to traditional PCA method [16]. Though PCA is widely used it suffers from immense computational cost, oversize storage requirement and low efficacy in dealing through huge dimensional data [17]. As we know that PCA is used for dimension reduction, but it is computationally expensive and does not eliminate anomalies that can be seen at one arbitrary band[10]. Here, small target are limited to the variance, that is, in much higher variance, smaller targets may not appear after PCA analysis, the issue was addressed in [18] using the ICA.

Majority of Significant information are extracted from data table, keeping only important information to reduce the size, if dataset explanation are made short, variables are structures are analyzed, these few steps followed by PCA[22]

\section{Independent Component Analysis (ICA)}

Independent-component analysis (ICA) is a popular technique for unsupervised classification[6]. ICA was first initiated in late 1980's and proposed in 1994 by Pierre Comon, and has been used in a enormous applications, including blind source separation, feature extraction, recognition etc[9]. ICA is a multivariate data analysis technique, it searches for a linear combination which reduces the statistical dependence between components. PCA, transform of experimental data is found, de-correlates the experimental variables during second-order statistics. Because of stronger, independent and uncorrelated components ICA uses higher order statistics to determine the projections of data[19]. ICA is skilled in classification, feature extraction and target detection in hyperspectral images[18]. Author tells that the majority target detection algorithms uses a priori obtainable target, target is, seldom available a priori. ICA is technique which aims at identifying the components which are statistically independent. Because of ICA

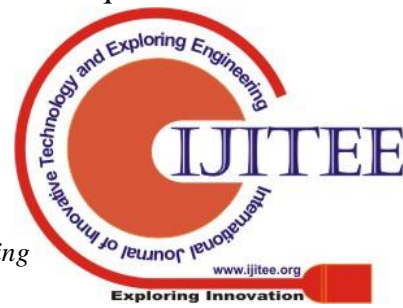


do not use prior information, this method has more potential for target detection application[5]. Because of classifying unknown spectral signature objects of unknown image, ICA became more popular. These leads to high computational complexity, high-dimensional data analysis and impede the application. So, in addition ICA classification is done after reducing data dimensionality by PCA[6]. And also, ICA dimensionality reduction is constructed using mutual information, whereas PCA dimensionality reduction is constructed using data variance \& SNR.

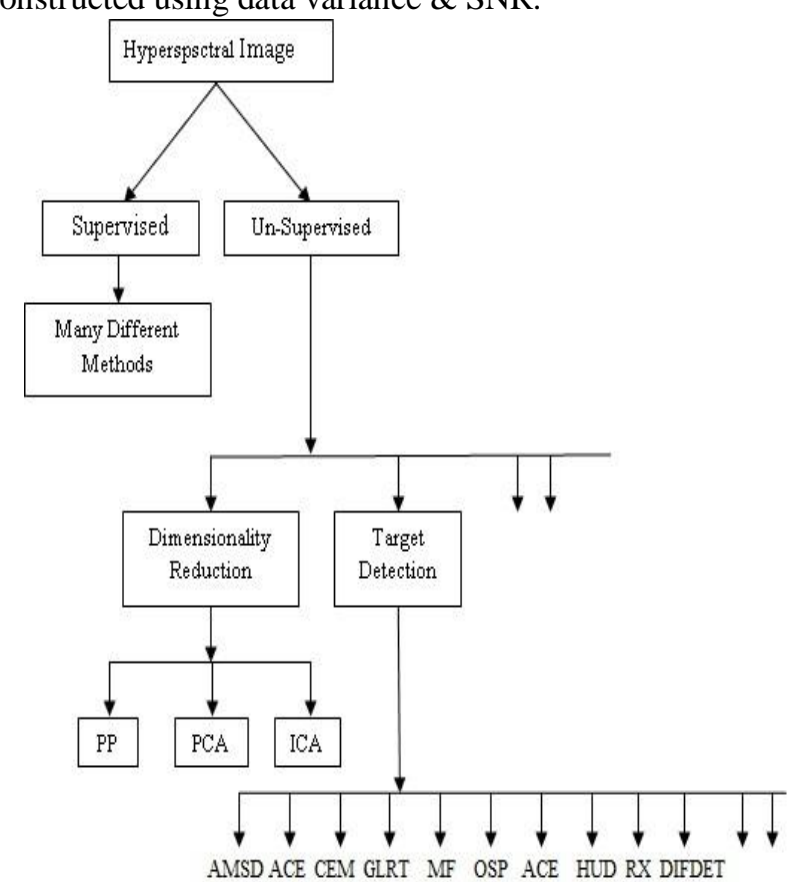

Fig.1: Classification of Hyperspectral Image processing Techniques.

Fig 1 shows the steps followed in review of target detection and dimensionality reduction in HSI processing.

\section{HYPERSPECTRAL IMAGE ANALYSIS CHALLENGES}

Using one panchromatic band or at three color bands (red, green, blue), earlier systems collected data, now hyperspectral bands are used [20], these analysis became challenge. The data possessed from the identical materials are distinct because of the variations in the composition of materials, noise of sensors and the propagation of atmosphere. The differences in the identical materials make the analysis of image very interesting.

The listed hurdles of hyperspectral remote sensing are below,

\section{> Processing and Visualization Problems \\ $>$ Handling Huge Data. \\ $>$ Data Redundancy \\ > The Dimensionality Problem}

\section{TARGET DETECTION}

Defense organization, mineral examination, search and rescue operations, border security, agricultural crops and several other anthropogenic and natural object/phenomenon and other applications essentially uses target detection technique were proposed over the decades, classification of target detection and analysis of them is done [1]. To find better algorithm, the following has done, First, the key issues involved in the design and evaluation of detection algorithms for hyperspectral imaging data are discussed. Second, a critical review of existing detection algorithms for practical hyperspectral imaging applications. Finally, argue that the "apparent" superiority of sophisticated algorithms with simulated data, by doing all this, matched filter and ACE detectors given good performance[7].

There are many algorithms which performs detection but, no algorithm guarantees about real data target, gives clear information and which should be merged. Apart from[1], eight signature-based hyperspectral target detection algorithms, namely Generalized Likelihood Ratio Test(GLRT), Adaptive Matched Subspace Detector(AMSD), Signed Adaptive Coherence Estimator(SACE) and Adaptive Coherence Estimator(ACE) (The adaptive algorithms operates relatively well with the difficulty of unidentified backgrounds[4]), Hybrid Unstructured Detector(HUD), Orthogonal Subspace Projection(OSP), Constrained Energy Minimization(CEM) and Matched Filter(MF), three anomaly detectors, namely RX, Maxmin and Diffdet, are been examined and compared. Along with signature-based target detectors, three finest performing algorithms which contain complementary information were recognized. At the end, these algorithms fused along with four different fusion algorithms[11].

Among above defined algorithms, SACE and CEM is better performance algorithm. AMSD declared as best performance algorithm, it performs best as target space of the sub pixel is nearer to the lower partial region of the pixel.Nevertheless, the AMSD demands for the back ground members to model (it's a pure material or a spectra and pure pixel that will be always identified as an end members with typical feature of spectral signature), which raises the computational complexity. All three algorithm given success and weakness on particular region, hence all were gathered using hybrid fusion technique [11].

It's been examined that the result of a CEM identification is comparatively indigent [3], the poor identification of lower level CEM deficiency issue is solved, by two excellent identification methods: first one is Principle Component CEM and second one is Matrix Taper CEM, along with these two an advanced Two Time identification method is designed. Originally, the significant target features are detected with MT CEM and PC CEM techniques. The identification efficiency of MT CEM and PC CEM methods differs with data of the image. These methods were not an robust identifier, the techniques that is able to yield an higher quality of target features identification result is better when compared to above methods is TTD technique.

The researcher in this work illustrates that no best Hyperspectral identification algorithm is designated for every object and image. The researcher determines that RBTA is employed to select the correct identifiers among various identifiers separated from background reality. Hence, they admitted the Improved RBTA, where the 
specified growth depends on the target feature size. They tested the designs at the time of selection for the best identifiers within a group of stochastic methods for the target feature identification, i.e. CEM, GLRT, and ACE predicts finest algorithms for a precise images and objects [21].

The researcher adapted the comparative method for target feature identification methods in HIS that are useful for situational crops in Colombia within the images that are acquired by Hyperspectral sensor satellite Hyperion. The algorithms examined were ACE, CEM, MF, SAM and OSP. The outcome demonstrate that the ACE algorithm has a improved performance followed by CEM and MF algorithms, whereas OSP and SAM fails due to more false alarms[12].

The target identification of a Hyperspectral images employing ICA techniques related to spectral designing are of tremendous interest[5]. The author matches up an ICA in addition to the alternatives four spectral images matching algorithms in specifically Constrained Energy Minimization, Orthogonal Subspace Projection, Spectral Correlation Mapper, Spectral Angle Mapper, along with different four more anomalies identification methods such as Reed Xiaoli Anomaly Detector, OSP anomaly Detector - OSPAD, Uniform target detector - RXDUTD were determined. The interpretation of false and true positive ratio of target features identification achieved by employing ICA along with additional methods designates better efficiency of ICA when compared to other additional algorithms[3].

\section{PROBLEM IDENTIFICATION}

Identifying theoretical computational problem in Hyperspectral image processing itself is a big problem. The theoretical computational difficulties are: Finding/Placing data in a specific format, dig into data, carry out analysis in High Performance Computing(HPC) way, remove sensor noise and atmospheric noise, identifying end-member / target. All this problems can be solved using parallel computing aspect while doing HPC.

\section{A. Methodology}

- $\quad$ ICA is used instead of PCA [24]-[25].

- Noise Reduction (removing high frequency noise by cloud cover, Atmospheric correction is an important pre-requisite step to enhance and improve identification of spectral signatures of different objects or materials and their compositions[8]).

- Data Spaces (Dimensionality reduction).How much of dimensionality reduction is needed? Data mining /data-cube concepts are used then no need to do data reduction. This is where computational skills come into picture to enter any part of the cube. A various dimension reduction techniques exist: PCA, minimum noise fraction, locally linear embedding independent component analysis , discrete wavelet transform etc[17].

- Data Mining (data reduction). algorithms. The demonstration shows that the concept

\section{LITERATURE GAP AND CONCLUSION}

All the Hyperspectral Image (HSI) Processing algorithms discussed were built on their own assumptions and thus have limitations. The fundamental approach and the extreme recognized research works are considered for discussion in addition the best current approaches in every visible feature of HS digital images processing. Both the most classic and advanced work were introduced. From this work, directions in current research trends are revealed [2].

Still not yet developed an general Hyperspectral Image Processing system. The method used for HSI processing should be a function problem type, Target Detection, Material Mapping, Material Identification, Mapping details and surface properties, Material Classification, Atmospheric correction etc. from remote sensing data.

To identify the target first we need to reduce the band size, from 224 band to suitable size of 10-20 bands, to perform this Independent Component Analysis is used instead of PCA, Next the Noise has to be Reduced( removing high frequency noise by cloud cover ). The reduction in dimensionality of the data space is performed through the appropriate choice in a cluster of data mining reduction methods, utilizing these methods; it's easy to diminish the dimensionality and redundancy of data, and in the extraction of particular features information from the Hyperspectral image [13].

Target Detection methods are not always consistent particularly for hyperspectral data. Hence we need to find the better target detectors. But it requires much effort. Fusion methods produce precise results.

It is also found that hybrid methods like Genetic algorithms, swarm techniques, Bayesian formulations [23] despite the hopeful research results, are not frequently used. Hybrid methods [23] can be tried with physical models. Novel approaches like hyperspectral imageries using neural network, machine learning techniques may give improvement in target detection.

Cost and complexity are the main draw-back. Computers with High-speed, responsive detectors, and massive records storage space are required for analyzing hyperspectral records. Considerable data storage capacity is essential because hyperspectral cubes are huge, multidimensional datasets, potentially greater than hundreds of megabytes. All of these factors significantly boost the cost of handing hyperspectral data.

\section{REFERENCES}

1. N.Poojary, Puttaswamy.MR, H D'Souza \& G.Hemanth Kumar, Automatic TD in HSIP: A review of algorithms, 2015 12th International Conference on Fuzzy Systems and Knowledge Discovery (FSKD), 978-1-4673-7682-2/15 2015 IEEE.

2. Zhang Liangpei and DU Bo, Recent advances in HSIP, Geo-spatial Information Science, Vol. 15, No. 3, September 2012, 143-156.

3. ZHENG Mao, ZAN Decai, ZHANG Wenxi, TD Algorithm in Hyperspectral Imagery Based on FastICA, 978-1-4244-5848-6/2010 IEEE.

4. D.Manolakis, D.Marden, and Gary A. Shaw, HSIP for Automatic TD Applications, VOLUME 14, NUMBER 1, 2003 LINCOLN LABORATORY JOURNAL.

5. K.C.Tiwari, M.K.Arora, D.Singh, An assessment of ICA for detection of military targets from HSI's, International 
Journal of Applied Earth Observation and Geoinformation, Volume 13, Issue 5, October 2011, Pages 730-740.

6. Qian Du, Ivica Kopriva, \& Harold Szu, ICA for HSRS imagery classification, Optical Engineering 45_1_, 017008 _January 2006_, (C) 2006 SPIE.

7. D. Manolakis, R. Lockwood, T. Cooley and J. Jacobson, Is There a Best HS Detection Algorithm?, Proc. of SPIE Vol. 7334, 733402.

8. N.Rani, VR.Mandla, T.Singh, Evaluation of atmospheric corrections on HS data with special reference to mineral mapping, Geoscience Frontiers(Elsevier) 8 (2017) 797e808.

9. Hongtao Du, DR using Parallel ICA in HSI Analysis, A Thesis Presented for the Master of Science Department of Electrical and Computer Engineering, The University of Tennessee, Knoxville, May 2003.

10. Kaewpijit.S, Moigne.JL., and El-Ghazawi T, Automatic reduction of HSIery using wavelet spectral analysis, IEEE Transactions on Geoscience and Remote Sensing, 41:863-871, 2003.

11. SE Yuksel \& A Karakaya, Fusion of TD Algorithms in HSI's, International Journal of Intelligent Systems and Applications in Engineering, Advanced Technology and Science ISSN:214767992147-6799.

12. A C Velasco, CAV García, \& HA Fuentes, A comparative study of TD algorithms in HSI applied to agricultural crops in Colombia, Tecnura • p-ISSN: 0123-921X • e-ISSN: 2248-7638 • Vol. 20 No. 49 - July - September 2016 • pp. 86-99.

13. G-Sreekala, Bajwa and SS. Kulkarni, HS Data Mining, HSRS Vegetation, A Huete, TS Prasad \& JG Lyon, , CRC Press 2011, Print ISBN: 978-1-4398-4537-0.

14. S-S Chiang, C-I Chang, and IW. Ginsberg, Unsupervised TD in HSI's Using PP, IEEE Transactions on Geo-science and Remote Sensing, vol. 39, NO. 7, July 2001.

15. JA. Malpica, JG. Rejas, MC. Alonso, A PP algorithm for anomaly detection in HSI, Elsevier Pattern-Recognition 41 (2008) 3313 3327, ISSN: 0031-3203.

16. A Agarwal, T El-Ghazawi, $\mathrm{H}$ El-Askary, and J Le-Moigne, Efficient Hierarchical-PCA DR for HSI, 2007 IEEE International Symposium on Signal Processing and Information Technology, 978-1 -4244-1 835-0/07, pp 353-356.

17. Deepa. P \& K. Thilagavathi, Feature Extraction of HSI Using PCA and Folded-PCA, IEEE Sponsored 2nd International Conference On Electronics And Communication System (icecs 2015), 978-1- 4788$7225-8 / 15$.

18. Robila, S. A., \& Varshney, P. K. TD in HSI's based on ICA. In AeroSense 2002 (pp. 173-182). International Society for Optics and Photonics.

19. RJ Johnson, Improved feature extraction, feature selection, and identification techniques that create a fast unsupervised HS TD algorithm, Thesis, Approved For Public Release; Distribution Unlimited, March 2008.

20. Shaw, GA., and Burke, H.-h. K. Spectral imaging for remote sensing. Lincoln Laboratory Journal 14, 1 (2003), 3-28.

21. Y Cohen, Y August, Dan G. Blumberg, and Stanley R. Rotman, Evaluating Subpixel TD Algorithms in HSI,. Hindawi Publishing Corporation Journal of Electrical and Computer Engineering, doi:10.1155/2012/103286.

22. Herve Abdi and Lynne J. Williams, Principal component analysis, 2010 John Wiley \& Sons, Inc. WIREs Comp Stat 20102 433-459.

23. Miguel A. Veganzones \& M Graña, Hybrid Computational Methods for HSI Analysis, E. Corchado et al. (Eds.): HAIS 2012, Part II, LNCS 7209, pp. 424- 435, 2012.

24. Ozan ARSLAN, Ozer AKY UREK \& S_inasi KAYA, A comparative analysis of classi_cation methods for hyperspectral images generated with conventional dimension reduction methods, Turk J Elec Eng \& Comp Sci (2017) 25: 58-72.

25. Xin Qin Nian Yongjian Li Xiu* Wan Jianwei Su Linghua**, Dimensionality Reduction for Hyperspectral Imagery Based on FastICA, JOURNAL OF ELECTRONICS (CHINA), Vol.26 No.6, November 2009.

\section{Author Profile}

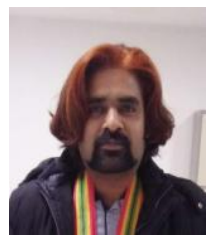

Kiran Gowda C received B.E degree in Computer science and engineering from MMEC, VTU in 2005 , M.Tech degree from NIE, VTU in 2013. Currently pursuing Ph.D in RRCE, VTU. His research interest are Hyperspectral remote sensing, image processing and machine learning. Currently he is working as Project Lead in R\&D Department, Infotop, Linyi. China.

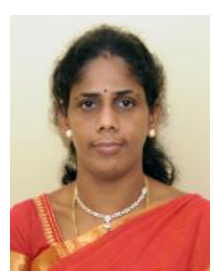

S. Usha, is currently working as a Professor and Head , CSE, RRCE with an experience of 21 years. Graduated from Manonmanium Sundaranar University, in Computer Science and Engineering during the year 1998. She obtained her Master degree in Computer Science and Engineering and $\mathrm{PhD}$ degree from sathyabama university in the area of Mobile Ad Hoc Networks in the year 2013. She has 54 publications in International and National conferences, 22 publication in national journal and international journals in the area of Mobile Ad hoc Networks and wireless security. Most of the publications are having impact factor citied in SCI, google scholar,scopus (h index and i10index), Microsoft etc.. Received fund from AICTE under NCP scheme ,MODROBS ,TGS and SERB(DST). Received best teacher award from Lions club in the year 2010\&2012.Received best paper award in many conferences. Developed Centre of Excellence lab in IoT with industry collobration.Organized many conferences ,FDPs and Technical Talks. Associated with ISTE, CSI,IEEE,IAENG ,IDES and IACSIT.Reviewed papers in IJCs and CiiT jouranals. Acted as a TPC member in MIRA'14 IoTBDS ' 17 and IoTBDS'18 Portugal.. Chaired sessions in FCS'14, ICISC'13 \& ICCCT'15., ICCCT'17and IoTBDS'18.Local chapter Active SPOC for NPTEL ,College website co-ordinator and NBA co-ordinator at college level.

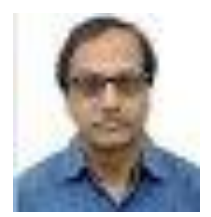

C J Jagadeesha received his B.E degree in civil engineering from KREC (now NITK) Surathkal in 1979 and M.E degree from Osmania University, Hyderabad in 1989. He will shortly receive Ph.D Award from KITS [Deemed to be University] Coimbatore. Currently he is working as a Adjunct Professor ,Department of Civil Engineering, KITS Coimbatore. He worked as a Scientist SE / Engineer, ISRO- NRSC- RRSC (S): Regional Remote Sensing Centre (South) in Bangalore, Karnataka, India. He is specialized in the areas of water resources, remote sensing, image processing and geoinformatics. He is a life member of various professional bodies like Indian Society of Remote Sensing, Indian Meteorological Society, Centre for Spatial Database Management, and a member of Bangalore Science Forum, Karnataka Environmental Research Foundation, Indian Water Resources Society, Institute for Climate Change and Sustainability (ICCS), Environmental Association of Bangalore, Bangalore, FIE ,IEI_KSC. He has 60 publications both in national, international journals, Workshops, Conferences and invited talks. 